\title{
A Method to Differentiate Pathological Murmurs from Innocent Murmurs in Cardiology
}

\author{
Jui-chien Hsieh ${ }^{1}$, Xiaomeng $\mathrm{Wu}^{2}$, Yi-Hsing Claire $\mathrm{Chiu}^{3}$ \\ ${ }^{1}$ Dept. of Information Management, Yuan Ze University \\ Taiwan \\ jchsieh@saturn.yzu.edu.tw \\ ${ }^{2}$ Dept. of Biomedical Engineering, Chongqing University \\ China \\ 1663871540@qq.com \\ ${ }^{3}$ Dept. of Applied Psychology, Hsuan Chuang University \\ Taiwan \\ cchiu@hcu.edu.tw
}

\section{Extended Abstract}

The technology of detecting cardiac valve related diseases using echo-ultrasonics is progressing. Nevertheless, the screening of cardiac valve diseases in rural clinics is mainly dependent on the auditory interpretation of heart sounds. There is a strong demand of developing a computer-assisted algorithm to help rural physicians distinguish abnormal heart sounds and normal heart sounds with innocent murmurs.

In this study, we examined 10 patients with normal heart sounds with innocent murmurs, and 12 patients with aortic stenosis, mitral regurgitation, of mitral stenosis, whose data was randomly collected using 3M Littmann digital stethoscope at Taoyuan Army Forced General Hospital in Taiwan from July to November in 2016 [1]. The heart sound data was preprocessed to minimize environmental noises and then processed with singular spectrum analysis (SSA), which separated murmurs from recorded heart sounds [2-4]. The power spectrum densities (PSD) of extracted murmurs in two groups were estimated. Results indicated that the patterns of PSD of extracted murmurs in normal and abnormal groups can be discriminated. In addition, a ratio, the area under curve from $201 \mathrm{~Hz}$ to $300 \mathrm{~Hz}$ to the area under curve from 0 to 200 $\mathrm{Hz}$ in PSD, seemed to be an effective index to discriminate abnormal and innocent murmurs with independent sample $\mathrm{T}$ test ( $\mathrm{p}$ value < 0.05). In conclusion, this developed algorithm can help physicians better differentiate abnormal murmurs and innocent murmurs, which is useful for the further development of phonocardiogram telemedicine.

\section{References}

[1] 3M stethoscope web portal, [Online]. Available: http://www.littmann.com/3M/en_US/littmann-stethoscopes

[2] S. Sanei, M. Ghodsi, H. Hassani, "An adaptive singular spectrum analysis approach to murmur detection from heart sounds," Medical engineering \& physics, vol. 33, no. 3, pp. 362-367, 2011.

[3] S. Sanei, H. Hassani, Singular spectrum analysis of biomedical signals. CRC Press, 2015.

[4] Y. Chen, S. Wang, C. H. Shen, F. K. Choy, "Matrix decomposition based feature extraction for murmur classification," Medical engineering \& physics, vol. 34, no. 6, pp. 756-761, 2012. 